\title{
Optical and Electron Microscopy of Au core/CdS Shell Nanoparticles
}

\author{
M. Nabil ${ }^{a}$, K. Easawi ${ }^{a}$, M.K.Elmancy ${ }^{c}$, S. Negm ${ }^{a}$, and H.Talaat ${ }^{b}$ \\ ${ }^{a}$ Department of Mathematical and Physical Engineering, Faculty of \\ Engineering (Shoubra), Banha University, Cairo, Egypt \\ ${ }^{b}$ Physics Department, Faculty of Science, Ain Shams University, \\ Abbassia, Cairo, Egypt \\ ${ }^{c}$ Physics Department, Faculty of Science, Benha University.
}

The combining of metal and semiconductor nanostructures as core/shell have attracted great interest due to their complementary optical properties; the localized Plasmon in the core and the exciton in semiconductor. The first, results in the enhancement of local fields and the second, are the nonradiative damping due to electron transfer from semiconductor to metal. Composite nanostructures of metal-core/semiconductor-shell configuration were prepared using organometalic method. UV-Vis spectra for the colloids show two distinct bands, one for the gold (Au) metal core surface plasmon at lower energy and the second for the excitons of semiconductor Cadmium Sulfide (CdS) shell at higher energy. Second derivative methods were used to determine the precise absorption peaks in UV-Vis spectra. The shell size was estimated using two methods, effective mass approximation (EMA), and polynomial fitting function $(P F F)$, both give shell thickness dimension in the range of few nanometers. These dimensions were confirmed by imaging the nanostructure using high resolution transmission electron microscope (HRTEM).

\section{Introduction}

Recently, the growing ability to fabricate Metal/Semiconductor (M/S) composites on a nanoscale has opened up new opportunities for designing multifunctional materials with properties that cannot be obtained in the bulk phase. Over the past decade, a wide variety of nanocomposite morphologies, including metal-core/semiconductor-shell heterostructures were synthesized [1-3]. The fundamental interaction between semiconductor (shell) excitons and surface plasmon (SP) of metal (core) nanoparticles is of particular interest. The ability to tailor the optical properties or tune the sensing properties of the semiconductor nanoshell is a major goal of designing these nanocomposite 
systems [4]. Core/shell $\mathrm{Au} / \mathrm{CdS}$ nanostructure are of special interest in light energy conversion systems. The surface plasmon of a metal nanostructure is dependent on both the nanostructure size and chemical surroundings [5]. In this work we have used nanostructure gold core of the same dimension and of CdS shell of varying thickness.

UV-Vis spectra for the nanocomposites show the CdS exciton peak where as the peak corresponding to the Au core plasmon is relatively quenched The surface plasmon field of the core enhancement the local field in the nanostructure. Organometallic synthesis method was used to control Au-core size and CdS shell thickness. The dimensions of nanostructure were determined using the effective mass approximation (EMA) and also polynomial fitting function (PFF).The dimension were also measured directly using high resolution transmission electron microscope (HRTEM), and the calculated dimension are in good agreement with the experimentally measured once.

\section{Experiential}

\subsection{Preparation of Au core Nanoparticles:}

Gold acetyl acetenate ( $\mathrm{Au}$ ac ac) was prepared by mixing $3 \mathrm{ml}$ ethyl alcohol and $0.1 \mathrm{gm}$ of Gold (III) chloride (HAuCl4, Aldrich), at room temperature, then raising the temperature slowly to $90{ }^{\circ} \mathrm{C}$, and adding $0.5 \mathrm{ml}$ of acetyl acetone (Alpha Chemical) till we obtain red color. The temperature was raised until the liquid evaporates to get $(\mathrm{Au} \mathrm{ac} \mathrm{ac})$ powder. $2 \mathrm{ml}$ di-phenyl ether (Riedel-Dehaen), $2 \mathrm{gm}$ of Hexadecylamine (HDA, Aldrich) and $2 \mathrm{gm}$ Trioctylphosphine oxide (TOPO, Fluka), are added to the prepared Au ac ac at temperature $90{ }^{\circ} \mathrm{C}$ and by raising the temperature to $100^{\circ} \mathrm{C}$, the $\mathrm{Au}$ nanoparticles is formed. The absorption spectra were measured using UV-Vis spectrophotometer (Jasco 670).

\subsection{Preparation of $\mathrm{Au} / \mathrm{CdS}$ core shell Nanoparticle:}

The prepared $\mathrm{Au}$ core was put in tri-neck flask and heated to $140{ }^{\circ} \mathrm{C}$. A $0.3 \mathrm{~g}$ of cadmium oxide (CdO, Aldrich), was dissolved in $2 \mathrm{~g}$ (stearic acid) at $170{ }^{\circ} \mathrm{C}$ then a mixture of $2 \mathrm{~g}$ of TOPO and $2 \mathrm{~g}$ of HDA was added and held at 180 ${ }^{\circ} \mathrm{C}$ for $5 \mathrm{~min}$ to get a clear solution. The resultant solution was kept warm and stored in a syringe to use as a cadmium (Cd) source. A $0.3 \mathrm{~g}$ of sulfur (S) was dissolved in $6 \mathrm{ml}$ of Trioctylphosphine (TOP, Fluka), then stored in another syringe to use as a S source. Then $1 \mathrm{ml}$ of the Cd source and $1 \mathrm{ml}$ of S source were added drop wise into the $\mathrm{Au}$ core colloids and we waiting for $1 \mathrm{~min}$ to collect the first sample. Repeating the previous steps after adding more $\mathrm{Cd}$ and $\mathrm{S}$ 
and waiting 1 min each injection to collect the samples. At constant temperature 4 samples were collected at increasing time intervals and labeled from 1 to 4 . All samples were immediately cooled and diluted with toluene to stop $\mathrm{CdS}$ shell growth. The absorption spectra were measured using UV-Vis spectrophotometer. The particle size and shape was determined using high-resolution transmission electron microscopy (HRTEM) (JEOL JEM-2100 operated at 200KV with high resolution Gatan CCD bottom camera (Orius SC200)).

Plasmon excitation in the gold nanoparticles occur around $524 \mathrm{~nm}$. The surface plasmon is caused by the oscillation of the core free electrons resonating with the incident light. The Au nanoparticles of the same batch were used as the seeds for the CdS shell nanocomposites .

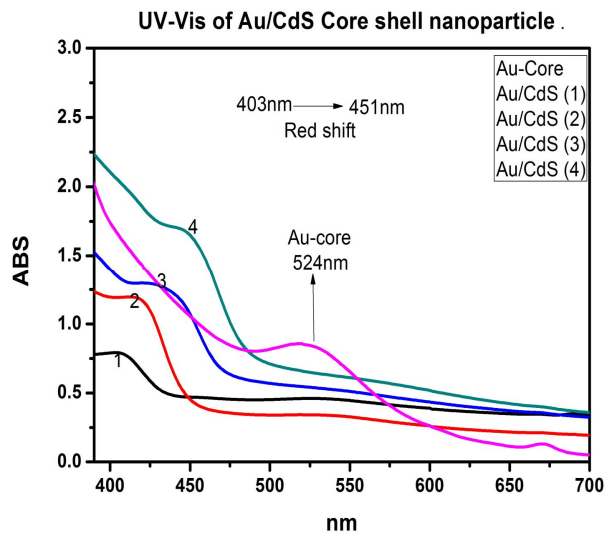

Fig. (1): UV-vis of $\mathrm{Au} / \mathrm{CdS}$ core shell nanoparticles

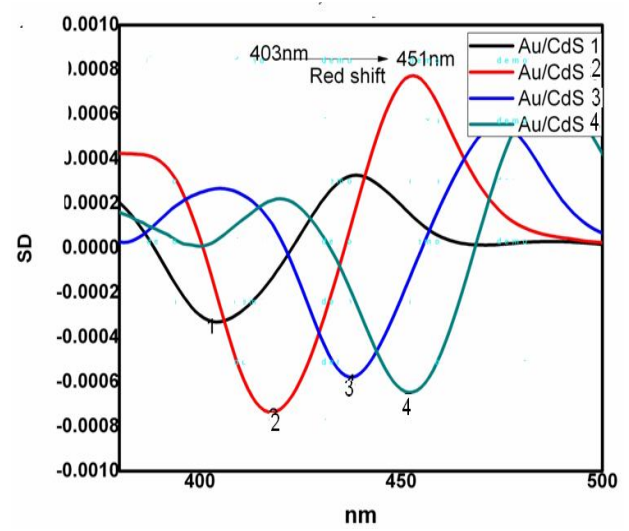

Fig. (2): Second derivative for CdS-shell spectra of $\mathrm{Au} / \mathrm{CdS}$ core shell nanoparticles .

There is an increase in intensity of the CdS shell exciton peak with increasing thickness. How ever, the peaks corresponding to the $\mathrm{Au}$ core show large decreases. The second derivative analysis was employed to the two regions, the plasmon and the exciton. The second derivative spectra, are given in Figs.(2) for the exciton of CdS shell region. There is a peak red shift of the excitonic band of CdS-shell from $403 \mathrm{~nm}$ to $451 \mathrm{~nm}$ as the shell thickness increase. There is also a red shift of the plasmon band of the gold core (about 30nm) as CdS shell increase. The red shift of the exciton can be explained in terms of the increasing of the shell width. On the other hand the red shift of the plasmonic absorption peak could be understood in terms of the loading of Au core with the increasing the shell thickness. 


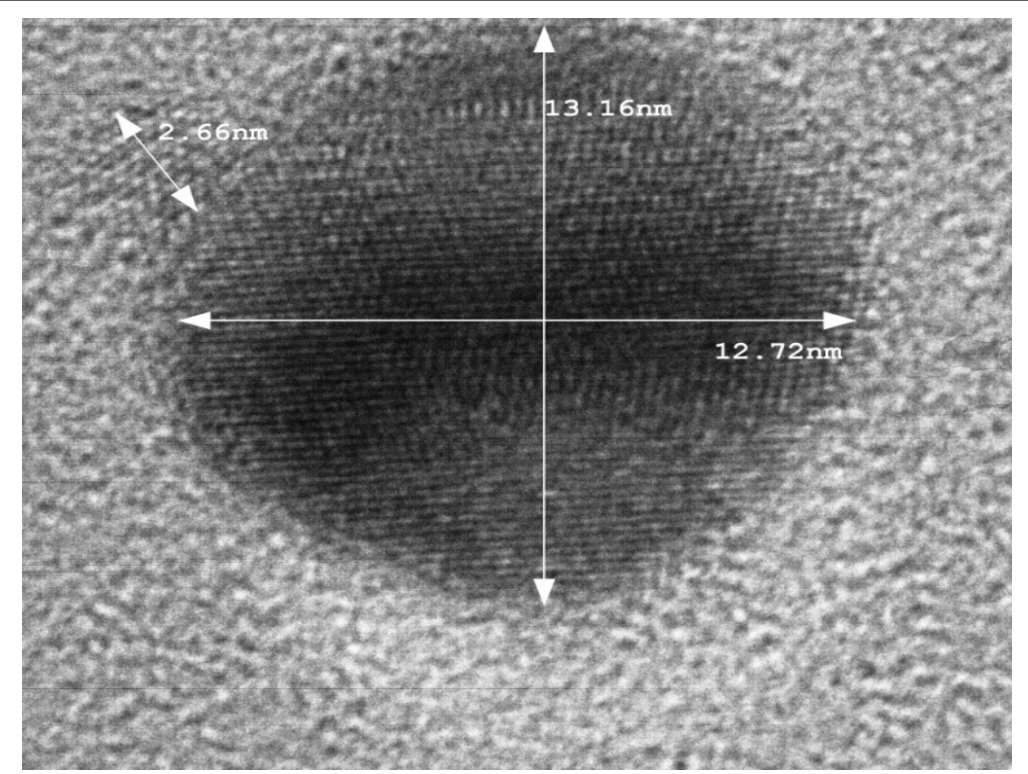

Fig (3): HRTEM of core/shell Au/CdS (Sample2) of $12.72 \mathrm{~nm}$ core and $2.66 \mathrm{~nm}$ shell thickness.

The size and structure of $\mathrm{Au} / \mathrm{CdS}$ were imaged by HRTEM, Fig. (3) presents the case for sample 2 in the optical spectra. It is clear that metallic gold nanoparicles have higher contrast than the semiconductor CdS shell. For this case, the core size is about $12.72 \mathrm{~nm}$, and the CdS shell size is about $2.66 \mathrm{~nm}$. The nanoparticles size was estimated from the UV-Vis absorption spectra peaks using two methods:

\section{(1) The Polynomial Fitting Functions (PFF) [6].}

$$
D=\left(-6.6524 \times 10^{-8}\right) \lambda^{3}+\left(1.9557 \times 10^{-4}\right) \lambda^{2}-\left(9.2352 \times 10^{-2}\right) \lambda+(13.29) \rightarrow(1)
$$

$\mathrm{D}(\mathrm{nm})$ is a size of a given nanocrystals sample, $\lambda_{(\mathrm{nm})}$ is the wave length of the first excitonic absorption peak.

\section{(2) The Effective Mass Approximation (EMA) [7].}

$$
E_{g n}=E_{g b}+\frac{\hbar^{2} \pi^{2}}{2 R^{2}}\left(\frac{1}{m^{*}{ }_{e}}+\frac{1}{m^{*}{ }_{h}}\right)-\frac{1.8 e^{2}}{\varepsilon R} \rightarrow \text { (2) }
$$

where $E_{g n}(\mathrm{NC})$ is the lowest energy for electronic transition for nanocrystals, $E_{g b}$ is the band gap of bulk $\mathrm{CdS}(2.42 \mathrm{ev})$, and $\mathrm{R}$ is the average radius of nanoparticles. $m^{*}{ }_{e}$ Is the effective mass of electron $(0.19 \mathrm{me}), m^{*}{ }_{h}$ is the 
effective mass of hole $(0.8 \mathrm{me}) \varepsilon$ is the dielectric constant for CdS nanocrystals. In Table (1) we show the dimension of CdS shell thickness as determined from the PPF and EMA methods. Also the table indicate the dimension as determine by HRTEM.

Table (1): The calculated $\mathrm{CdS}$ shell thickness in $\mathrm{Au} / \mathrm{CdS}$ core/shell nanoparticles by two methods (PFF) and (EMA) from the optical spectra of UV-Vis and then the shell thickness as measured by (HRTEM).

\begin{tabular}{|l|l|l|l|}
\hline \multirow{2}{*}{ Samples } & PFF(nm) & EMA(nm) & \multirow{2}{*}{ HRTEM(nm) } \\
\cline { 2 - 3 } & UV-Vis & UV-Vis & \\
\hline Au/CdS 1 & 1.8 & 1.92 & - \\
\hline Au/CdS 2 & 1.96 & 2.1 & 2.66 \\
\hline Au/CdS 3 & 2.36 & 2.4 & - \\
\hline Au/CdS 4 & 2.68 & 2.75 & 3.49 \\
\hline
\end{tabular}

\section{Conclusion:}

We present a new approach for synthesis of $\mathrm{Au} / \mathrm{CdS}$ by directly assembling $\mathrm{CdS}$ of different shell thickness on same size Au core nanoparticles . The calculation methods using EMA and PFF give dimensions of CdS shell in good agreement with the directly measured dimension using HRTEM. It is concluded that increasing the $\mathrm{CdS}$ shell in the $\mathrm{Au} / \mathrm{CdS}$ core/shell nanoparticles gives us the ability to tune the band gap of the nanoparticles to harvest a wide range of visible solar spectra.

\section{Acknowledgment}

The Support of the Science and Technology Development Fund (STDF) Grant ID 377 is greatly appreciated.

\section{Reference:}

1. Zhang, J. Tang, Y. lee, K. Ouyang, M. Science, 327, 1634 (2010).

2. Shi. W.L. Zeng, H. Sahoo, Y. Ohulchannnnskyy, T.Y. Ding, Y. Wang, Z. L. Swihart, M. Prasad, P.N. Naaaaaaano lett. 6, 875 (2006).

3. Kim. H. Achermann, M. Balet, L.P. Hollingsworth, J.A. Klimov and V. I. J. am., Chem, Soc., 127, 544 (2005).

4. Yanfei Wang, Minjie Li, Spectrochimic ta Part a Ac A 64, 101 (2006).

5. C. Zhong, M. M. Maye, Adv. Mater. 13, 1507 (2001).

6. A. M. Okasha, Mona B. Mohamed, T. Abdallah, Anis B Basily, S.Negm, Talaat. Journal of Physics: Conference Series, 214, 012131 (2010). 
7. Brus, L. E.; "Electronic Wave-Functions in Semiconductor Clusters Experiment and Theory". J. Phys Chem., 90, 2555 (1986). 